\title{
Gene expression profile comparison between colorectal cancer and adjacent normal tissues
}

\author{
QIAN YANG ${ }^{1}$, MAOHUI FENG $^{2}$, XIANG MA ${ }^{2}, \mathrm{HUACHI} \mathrm{LI}^{2}$ and WEI XIE ${ }^{2}$ \\ ${ }^{1}$ Department of Ultrasound, Hubei Cancer Hospital; ${ }^{2}$ Department of Oncology, \\ Zhongnan Hospital of Wuhan University, Wuhan, Hubei 430071, P.R. China
}

Received July 27, 2015; Accepted October 13, 2016

DOI: 10.3892/ol.2017.6915

\begin{abstract}
The present study aimed to compare gene expression profiles between colorectal cancer and adjacent normal tissues, and to perform a preliminarily analysis of the key genes and underlying molecular mechanisms implicated in colorectal cancer development. Gene expression microarray chips were used to screen genes that were differently expressed between colorectal cancer and adjacent normal tissues. Approximately 1,183 genes were differentially expressed in cancer tissues compared with adjacent normal tissues $(\mathrm{P} \leq 0.05$; fold difference, $>2.0$ ), of which 570 genes were upregulated and 613 genes were downregulated. In total, 6 upregulated genes, including keratin 23 , collagen type X $\alpha 1$, collagen type XI $\alpha 1$, cell migration-inducing hyaluronan-binding protein, transforming growth factor- $\beta 1$ and V-Myc avian myelocytomatosis viral oncogene homolog, and 2 downregulated genes, including channel $\alpha$ subunit 7 and EPH receptor A7, were selected and validated using reverse transcription-quantitative polymerase chain reaction, which exhibited results that were consistent with the microarray analysis. These 1,183 differentially expressed genes were further classified into 71 groups based on their functions using gene ontology and pathway analyses. Kyoto Encyclopedia of Genes and Genomes analysis of these upregulated or downregulated genes suggested that 23 signaling pathways were involved. The present study preliminarily screened for and identified key genes and signaling pathways that may be closely associated with colorectal cancer development. However, subsequent gene function studies are required to verify these findings.
\end{abstract}

\section{Introduction}

Colorectal cancer (CRC) is a common gastrointestinal cancer and its global incidence is outranked only by gastric and

Correspondence to: Professor Wei Xie, Department of Oncology, Zhongnan Hospital of Wuhan University, 169 Donghu Road, Wuchang, Wuhan, Hubei 430071, P.R. China

E-mail: fengmh5690@163.com

Key words: colorectal cancer, complementary DNA, microarray, gene oncology analysis, pathway analysis esophageal cancers (1). There were 14.1 million new cases of cancer globally in 2012, of which CRC accounted for 1.4 million and in China, was surpassed only by lung and breast cancers (2). In China, the incidence of CRC and its mortality rate have been ranked third and fourth, respectively among malignant tumors (3). Studies have demonstrated that China's annual incidence of CRC increases twice as fast compared with the global average $(3,4)$. In Shanghai, Beijing and Guangzhou, the incidence of colorectal cancer is 40/100,000 individuals (5); and a previous CRC survey program in Wuhan, Hubei province has reported a high incidence of 90/100,000 individuals, which is twice that of the national average (6). According to the National Bowel Cancer Screening Programme, whose aim was to reduce the incidence of symptomatic CRC, the majority of patients present with symptoms to their general practitioner (7-9). An isolated symptom may be associated with CRC, but typically symptoms were observed in clusters. In total $\geq 1$ high-risk symptoms are seen in $\sim 85 \%$ of patients with CRC, who were referred to secondary care (10). However, symptoms for disease may be biased to a certain extent by 'selection phenomena', and this is the case to CRC (11). When performing diagnostic research in secondary care, this bias is not always considered (12). Additionally, the primary symptoms in the primary care setting may differ amongst the community and the hospital practice (11). Despite this being investigated by previous studies $(12,13)$, no significant molecular markers with a certain levels of efficacy or specificity for the early diagnosis of CRC have been identified. Therefore, further studies focusing on the molecular mechanisms of CRC development are imperative to identify molecular markers for the early detection of CRC and to develop individualized therapy, which would in turn improve the survival rate of patients with CRC. Based on high-throughput microarray technology, the present study aimed to identify potential key molecules as biomarkers in colorectal cancer development.

\section{Patients and methods}

Study subjects and tissue samples. In total, 6 patients with colorectal cancer, who were admitted to the Abdominal Oncology Department of Zhongnan Hospital of Wuhan University (Wuhan, China) between June 2012 and November 2012, were enrolled in this study. Colorectal cancer 
tissues and adjacent normal tissues were isolated intraoperatively, then immediately placed into prepared cryogenic vials, and frozen in liquid nitrogen within 5 min to avoid RNA degradation. The selection criteria of the case were: Diagnosis of colorectal cancer by postoperative pathological examination; and no preoperative radiotherapy, chemotherapy or other antitumor therapy. The present study was approved by Zhongnan Hospital of Wuhan University (approval no. 2010017), and written informed consent was obtained from all participants. The study was conducted in compliance with the Declaration of Helsinki.

Microarray chips. Affymetrix Human U133 Plus 2.0 chips (Shanghai Biotechnology Corporation, Shanghai, China) were used, which consisted of 47,000 transcripts, representing 38,500 known human genes.

Tissue RNA extraction, purification and quality control. Total RNA was isolated from the colorectal cancer and normal adjacent tissues using TRIzol (cat. no. 15596-026; Thermo Fisher Scientific, Inc., Waltham, MA, USA) by a 1-step extraction procedure, and the quality of the RNA was then examined using the Agilent Bioanalyzer 2100 (Agilent Technologies, Inc., Santa Clara, CA, USA) to perform electrophoresis. The acceptable quality threshold was set as an RNA integrity number (RIN) of $\geq 7.0$ and a $28 \mathrm{~S} / 18 \mathrm{~S}$ ribosomal RNA (rRNA) ratio of $>0.7$. To avoid a reduction in RNA purity, which may affect hybridization to the probe, total RNA was purified using the RNeasy Micro kit (cat. no. 74004; Qiagen GmbH, Hilden, Germany) and RNase-Free DNase set (cat. no. 79254; Qiagen $\mathrm{GmbH}$ ), according to the manufacturer's protocol (the RNeasy Mini Protocol for RNA Cleanup; Qiagen $\mathrm{GmbH}$ ).

Microarray assay. The purified total RNA sample was amplified and labeled using an Affymetrix microarray accessory GeneChip $^{\circledR}$ 3'IVT Express kit (Affymetrix, Inc., Santa Clara, CA, USA) to obtain biotin-labeled complementary RNA. The labeling efficiency and quality monitoring were performed by determination of fluorescent labeling efficiency to ensure the reliability of the subsequent microarray assay results. With the supporting standard process for hybridization and GeneChip ${ }^{\circledR}$ Hybridization, Wash and Stain kits (Affymetrix, Inc.) were provided along with the aforementioned Affymetrix microarray chips. Hybridization was conducted in a GeneChip ${ }^{\circledR}$ Hybridization Oven 645 (Affymetrix, Inc.) at $45^{\circ} \mathrm{C}$ for $16 \mathrm{~h}$. Subsequent to hybridization, the Fluidics Station 450 (Affymetrix, Inc.) was used to wash the chips in accordance with the manufacturer's protocol. Microarray results were scanned using the GeneChip ${ }^{\circledR}$ Scanner 3000 (Affymetrix, Inc.). Raw data were read using Command Console Software 3.1 (Affymetrix, Inc.) and qualified data were normalized using Gene Spring Software 11.0 (Agilent Technologies, Inc.). The algorithm used was MAS 5.0 (14). By analyzing and comparing the data in adjacent tissues and paired normal tissues, candidate marker genes associated with the occurrence of colorectal cancer were identified.

Reverse transcription-quantitative polymerase chain reaction $(R T-q P C R)$ to validate the microarray results. The top 4 upregulated genes, including keratin 23 (KRT23), collagen
Table I. Genes selected for reverse transcription quantitativepolymerase chain reaction validation.

\begin{tabular}{lcc}
\hline Gene & Fold change & P-value \\
\hline Upregulated genes & & \\
KRT23 & 111.37 & $8.57 \mathrm{e}-06$ \\
COL10A1 & 49.52 & $8.37 \mathrm{e}-05$ \\
COL11A1 & 48.54 & 48.54 \\
KIAA1199 & 39.46 & $4.55 \mathrm{e}-06$ \\
TGFBI & 4.41 & $7.15 \mathrm{e}-05$ \\
MYC & 3.19 & 0.008739 \\
Downregulated genes & & \\
SCN7A & -35.71 & 0.001 \\
EPHA7 & -30.30 & 0.008 \\
\hline
\end{tabular}

KRT23, keratin 23 (histone deacetylase inducible); COL10A1, collagen type X $\alpha 1$; COL11A1, collagen type XI $\alpha 1$; KIAA1199, cell migration-inducing hyaluronan-binding protein; TGFBI, transforming growth factor- $\beta 1$; MYC, avian myelocytomatosis viral oncogene homolog; SCN7A, sodium voltage-gated channel $\alpha$ subunit 7; EPHA7, EPH receptor A7.

type $\mathrm{X} \alpha 1$ (COL10A1), collagen type XI $\alpha 1$ (COL11A1) and cell migration-inducing hyaluronan-binding protein (KIAA1199), and the top 2 downregulated genes, including sodium voltage-gated channel $\alpha$ subunit 7 (SCN7A) and EPH receptor A7 (EPHA7), were selected for RT-qPCR validation. Transforming growth factor $\beta 1$ (TGFB1) and V-Myc avian myelocytomatosis viral oncogene homolog (MYC), which are established upregulated genes that demonstrate a clear association with cancer development (15), were used as positive controls. The basic information of the genes selected for qPCR validation is summarized in Table I. To synthesize first strand cDNA, the RNA was removed from storage in an $-80^{\circ} \mathrm{C}$ freezer and thawed at $4^{\circ} \mathrm{C}$. A reaction system (20 $\mu \mathrm{l}$ ) was then prepared using 4- $\mu 15 \mathrm{X}$ reaction buffer, $1-\mu 1$ RiboLock $^{\mathrm{TM}}$ RNase Inhibitor (Thermo Fisher Scientific, Inc.), $1-\mu$ RevertAid $^{\mathrm{TM}}$ M-Mu1 V Reverse Transcriptase (200 U/ $\mu$; Thermo Fisher Scientific, Inc.), 2- $\mu 110 \mathrm{mM}$ dNTP mix, 1- $\mu 1$ Oligo deoxy-thymidine 18 , and $2-\mu$ g RNA template; this was made up to $20 \mu \mathrm{l}$ with water. The PCR tubes were incubated at $42^{\circ} \mathrm{C}$ for $60 \mathrm{~min}$, denatured at $70^{\circ} \mathrm{C}$ for $5 \mathrm{~min}$ and stored at $-20^{\circ} \mathrm{C}$. Based on gene sequences in the Genebank database (https://www.ncbi.nlm.nih.gov/genbank/), primers were designed using Primer Premier 5.0 software (Premier Biosoft International, Palo Alto, CA, USA). Primers were synthesized by Shanghai Shenggong Biology Engineering Technology Service, Ltd. (Shanghai, China), and $\beta$-actin was used as an internal reference gene. Primer information is available in Table II. In total, 5- $\mu 1$ 2X SYBR-Green PCR buffer (Applied Biosystems; Thermo Fisher Scientific, Inc.), 0.2- $\mu 1$ forward primer $(10 \mu \mathrm{mol}), 0.2-\mu 1$ reverse primer $(10 \mu \mathrm{mol})$ and $5-\mathrm{ng}$ template were used for qPCR, and these were then made up to $10 \mu 1$ with water. The PCR cycling conditions were as follows: Incubation at $50^{\circ} \mathrm{C}$ for $2 \mathrm{~min}$, then $95^{\circ} \mathrm{C}$ for $10 \mathrm{~min}$, followed by 40 cycles at $95^{\circ} \mathrm{C}$ for $15 \mathrm{sec}$ and finally at $60^{\circ} \mathrm{C}$ for $1 \mathrm{~min}$. The ABI 7900 HT Sequence Detection System was used for 
Table II. Primer information.

Gene

KRT23

COL10A1

COL11A1

KIAA1199

TGFBI

MYC

SCN7A

EPHA7

$\beta$-actin
Primer sequences 5'-3'
F: AGTGAAGGGACACGGGAAGA

R: CCTGGGTTATGGCCTTGATCT

F: TCTCTAACTCTACCCCACCCTACAA

R: TACGTTTTTACGTTGCTGCTCACT

F: ACGCTGCATATACAGGTACCATTTAG

R: TCAGCCCTGTTTCCATCTTAGC

F: TCTAATGCAAGGGTCTCACACTGT

R: TGAACTGAGCCAAAGACATTCAA

F: GAGGAGGGAGAGAGATGTACTTTTTAAA

R: GGCAGTGACATCCAAGTTTCTG

F: CAAGAGGCGAACACACAACGT

R: AGGGCAAAAAAGCTCCGTTT

F: CATGTTATGGAGACCAGTGAGGAA

R: CCAAGAAATAGAAAACGGAGCTTAGA

F: CCTTGCTTTAATAGAGCCACCTTT

R: GGACCAGATCAATTGCTGAGAAA

F: CTGGAACGGTGAAGGTGACA

R: CGGCCACATTGTGAACTTTG

KRT23, keratin 23 (histone deacetylase inducible); COL10A1, collagen type X $\alpha 1$; COL11A1, collagen type XI $\alpha 1$; KIAA1199, cell migration-inducing hyaluronan-binding protein; TGFBI, transforming growth factor- $\beta 1$; MYC, avian myelocytomatosis viral oncogene homolog; SCN7A, sodium voltage-gated channel $\alpha$ subunit 7; EPHA7, EPH receptor A7; F, forward; R, reverse.

qPCR and SDS 2.3 software (Applied Biosystems; Thermo Fisher Scientific, Inc.) was used to analyze the data. The relative amount of the target gene in each sample was calculated using the $2^{-\Delta \Delta \mathrm{Cq}}$ method (16). The final results were generated from the data of two independent experiments.

Statistical analysis. Data analysis involved statistical analysis of microarray data and differentially expressed genes, cluster analysis, and gene ontology (GO) and pathway analysis. Microarray data analysis was performed using SAM 4.0 software (Stanford University, Stanford, CA, USA). Fold-changes (FC) of $>2.0$ or $<0.5$ (i.e., subsequent to calculation, genes with FC $>2$ were screened out) and $\mathrm{P} \leq 0.05$ were used as the criteria for selection of significant differentially expressed genes. In total $\sim 1,183$ genes that were differently expressed between cancer and adjacent cancer tissues were clustered using the unsupervised hierarchical clustering method in Cluster software (17) to generate clustering heat-maps in Treeview software (18). The heat-maps were then used to identify gene expression patterns associated with the samples. Based on GO (http://geneontology. org/page/go-database) and Kyoto Encyclopedia of Genes and Genomes (KEGG) database (http://www.genome.jp/kegg/) resources, differentially expressed genes were subjected to GO and pathway analyses using an enriched P-value algorithm, the R-package Fisher's exact test. $\mathrm{P}<0.05$ was considered to indicate a statistically significant difference.

\section{Results}

Baseline and demographic characteristics of patients. All 6 patients met the selection criteria; they consisted of
5 patients with colon cancer and 1 patient with rectal cancer (Table III). There were 4 male and 2 female patients. Each sample was assigned a hospitalization number, of which the middle 2 digits were concealed.

Quality control results of 12 RNA samples. The quality of the extracted total RNA was examined by Agilent Bioanalyzer 2100-mediated electrophoresis (Fig. 1). All of the samples exhibited RIN values of $\geq 7.0$ and $28 \mathrm{~S} / 18 \mathrm{~S}$ rRNA ratios of $>0.7$, indicating the RNA samples met the acceptable threshold.

Differentially expressed genes in colorectal cancer and adjacent cancer tissues. By comparing gene expression profiles of colorectal cancer and adjacent normal tissues, 1-class capabilities in SAM software filtered out 3,680 differentially expressed genes $(\mathrm{P} \leq 0.05)$, of which 2,043 genes were upregulated and 1,637 genes were downregulated in cancer tissues compared with normal tissues. To further screen for genes that showed significant differences in their expression, a FC of $>2.0$ or $<0.5$ was added as the standard to perform a second screen, which ultimately identified 1,183 differentially expressed genes, including 570 genes upregulated and 613 genes downregulated in cancer tissues compared with normal tissues. The raw chip data were standardized and $\log 2$-transformed, which gave rise to a scatter plot of the 1,183 differentially expressed genes (Fig. 2). A diagram of each point (representing the probe point on the chip) was generated, wherein the $\mathrm{x}$-axis represents the data of carcinoma tissues and $y$-axis represents the data of the adjacent normal tissues. In Fig. 2, points falling outside of the $45^{\circ}$ median lines 
Table III. Baseline and demographic characteristics of patients.

\begin{tabular}{lcccl}
\hline Sample no. & $\begin{array}{c}\text { Sample } \\
\text { name }\end{array}$ & Gender & $\begin{array}{c}\text { Age } \\
\text { (years) }\end{array}$ & $\begin{array}{c}\text { Lesion } \\
\text { site }\end{array}$ \\
\hline Cancer tissue & & & & \\
1 & $72^{* *} 10 \mathrm{Ca}$ & $\mathrm{M}$ & 64 & Colon \\
2 & $72^{* *} 62 \mathrm{Ca}$ & $\mathrm{M}$ & 68 & Colon \\
3 & $72^{* *} 34 \mathrm{Ca}$ & $\mathrm{F}$ & 74 & Colon \\
4 & $72^{* *} 75 \mathrm{Ca}$ & $\mathrm{M}$ & 64 & Colon \\
5 & $70^{* *} 39 \mathrm{Ca}$ & $\mathrm{F}$ & 40 & Rectum \\
6 & $70^{* *} 84 \mathrm{Ca}$ & $\mathrm{M}$ & 60 & Colon \\
Adjacent cancer & & & & \\
tissue & & & & \\
7 & $72^{* *} 10 \mathrm{~N}$ & $\mathrm{M}$ & 64 & Colon \\
8 & $72^{* *} 62 \mathrm{~N}$ & $\mathrm{M}$ & 68 & Colon \\
9 & $72^{* *} 34 \mathrm{~N}$ & $\mathrm{~F}$ & 74 & Colon \\
10 & $72^{* *} 75 \mathrm{~N}$ & $\mathrm{M}$ & 64 & Colon \\
11 & $70^{* *} 39 \mathrm{~N}$ & $\mathrm{~F}$ & 40 & Rectum \\
12 & $70^{* *} 84 \mathrm{~N}$ & $\mathrm{M}$ & 60 & Colon \\
\hline
\end{tabular}

M, male; F, female.

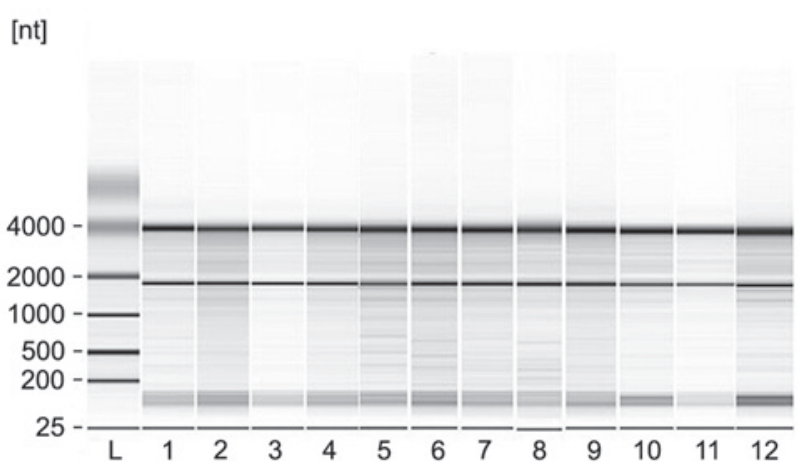

Figure 1. Denaturing agarose gel electrophoresis was performed to assess the quality of the RNA samples from colorectal cancer and adjacent normal tissues. L, ladder; 1-6, total RNA from 6 colorectal cancers; 7-12, total RNA from 6 adjacent normal tissues.

represent probe signal values where the average FC was either $>2.0$ or $<0.5$.

Validation of microarray results by RT-qPCR. Data obtained from microarray assays contain a fluorescent signal intensity of numerous noise points and missing values, causing a certain proportion of false positive and false negative results (19). Therefore, in order to verify the accuracy of the gene expression prolife chips, RT-qPCR analysis of the same samples was performed to evaluate the data quality of the microarray. The top 4 upregulated genes (KRT23, COL10A1, COL11A1 and KIAA1199) and the top 2 downregulated genes (SCN7A and EPHA7) in cancer tissues were selected for RT-qPCR validation. TGFBI and MYC, which are known upregulated genes in cancer tissues, were used as positive controls. As shown in Fig. 3, the results of the RT-qPCR were consistent with the microarray assay; genes

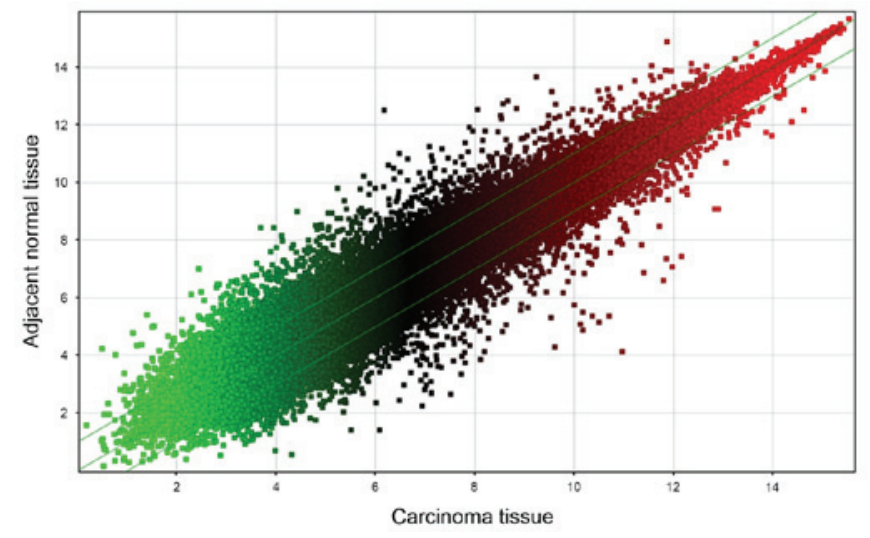

Figure 2. Scatter plot of differentially expressed genes. Green, reference gene; red; sample gene.

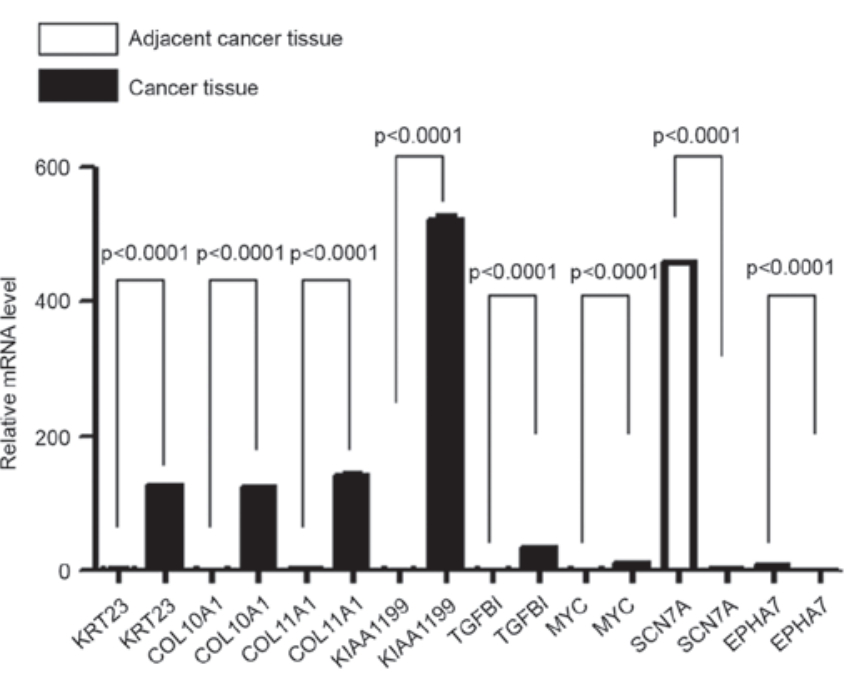

Figure 3. Reverse transcription-quantitative polymerase chain reaction results showing fold changes of selected differentially expressed genes.

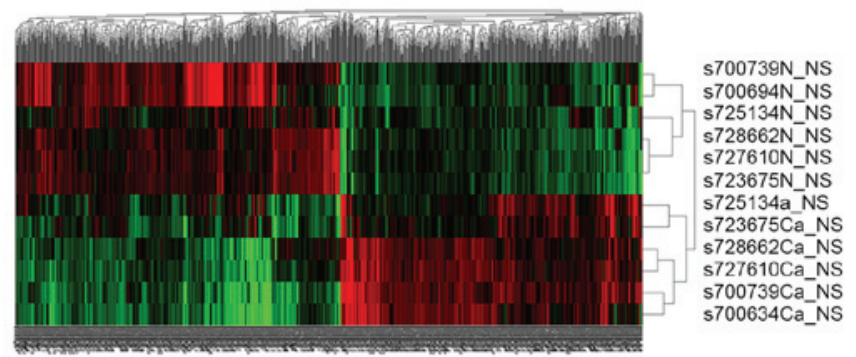

ㅇำ

Figure 4. Clustering analysis of the differentially expressed genes. Green, downregulated genes; red, upregulated genes.

that were upregulated in colorectal cancer tissues in the microarray results also showed upregulated expression in the RT-qPCR, while genes that were downregulated in the microarray results also showed downregulated expression in RT-qPCR. Therefore, it is considered that these 8 genes were differentially expressed between colorectal cancer and adjacent normal tissues. 

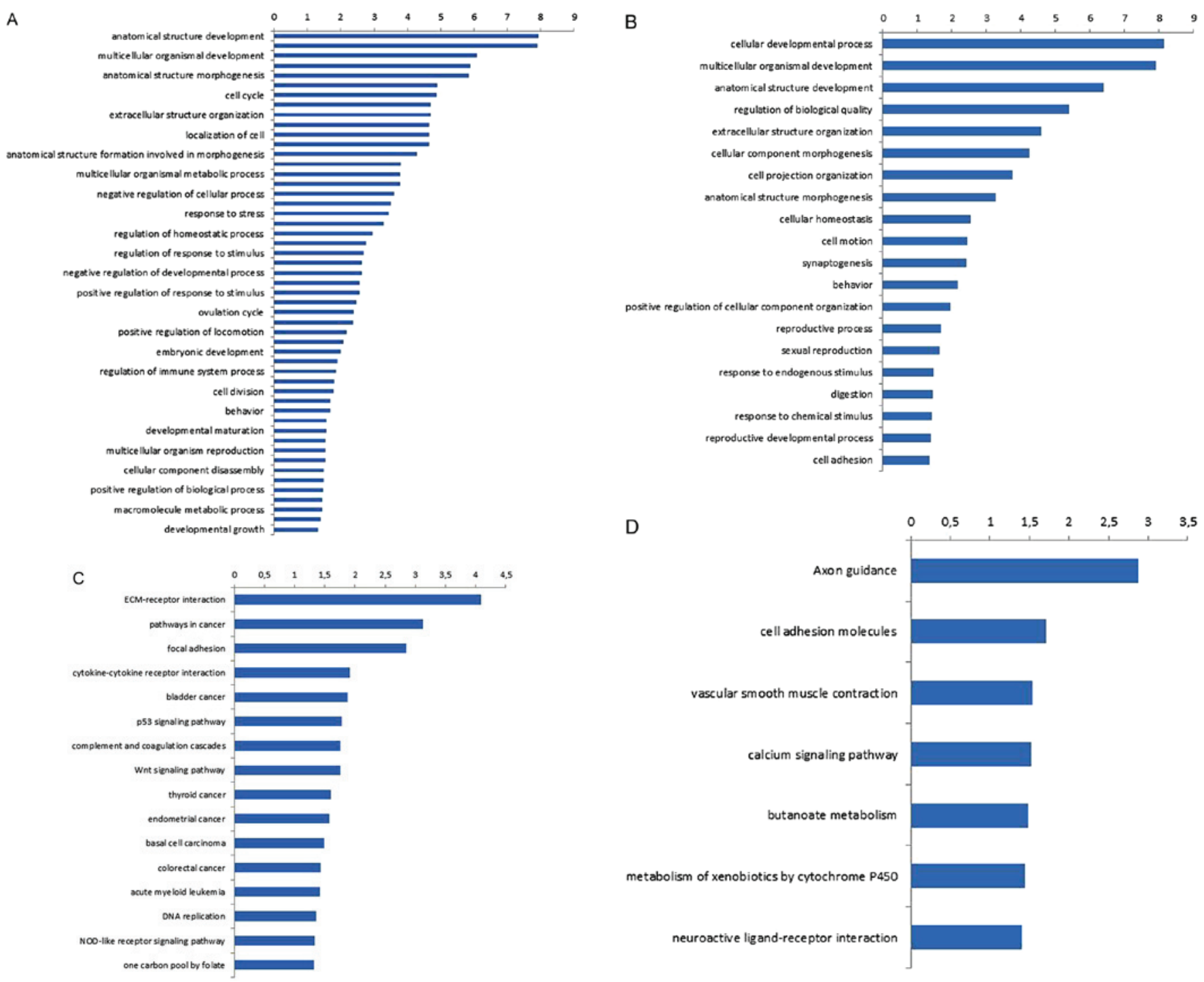

Figure 5. GO and KEGG pathway analysis of the differentially expressed genes. (A) GO analysis of the upregulated genes. (B) GO analysis of downregulated genes. (C) KEGG analysis of the upregulated genes. (D) KEGG analysis of downregulated genes. GO, gene ontology; KEGG, Kyoto Encyclopedia of Genes and Genomes.

Clustering, GO and pathway analysis of the differentially expressed genes. As exhibited in the clustered heat-map of the microarray analysis (Fig. 4), the 1,183 differentially expressed genes between cancer and adjacent normal tissues were divided into 2 groups using Clustering analysis. In Fig. 4, the abscissa represents the 1,183 differentially expressed genes, whereas the ordinate represent the 6 samples each of cancer and adjacent normal tissues from the bottom to the top. The red represents upregulated genes, whilst green represents downregulated genes; genes that exhibited no difference are shown in black.

GO analysis of the differentially expressed genes between the two groups of samples was performed to gain a preliminary understanding of the main biological functions. First, based on the GO database, the functions of these 1,183 differentially expressed genes were annotated, followed by calculation of the P-value and false discovery rate (FDR) for each GO term using Fisher's exact and $\chi^{2}$ tests. Finally, according to the screening criteria of $\mathrm{P} \leq 0.05$ and FDR $\leq 5 \%, \mathrm{GO}$ analysis results were obtained (Fig. 5A and B). As shown in the figure, according to the functions, the upregulated genes were divided into 51 categories, and the top 5 enriched $\mathrm{GO}$ function entries were: i) Anatomical structure development (99 genes); ii) response to external stimulus (50 genes); iii) multicellular organismal development (102 genes); iv) cell adhesion (38 genes); and v) anatomical structure morphogenesis (54 genes). The downregulated genes were divided into 20 categories, and the top 5 enriched GO entries were: i) Cellular developmental process (10 genes); ii) multicellular organismal development (18 genes); iii) anatomical structure development (13 genes); iv) regulation of biological quality (13 genes); and v) extracellular structure organization (5 genes).

A similar result was observed (Fig. 5C and D) using the KEGG database, wherein differentially expressed genes were annotated with functions to obtain all involved pathways. The P-value and FDR for each enriched KEGG term were calculated using Fisher's exact and $\chi^{2}$ tests, respectively. Subsequent to screening with the criteria of $\mathrm{P} \leq 0.05$ and FDR $\leq 5 \%, 16$ signaling pathways were shown to be associated with the upregulated genes, and the top 5 significantly enriched 
Table IV. Partial KEGG analysis results for the differentially expressed genes.

\begin{tabular}{|c|c|c|}
\hline KEGG entries & No. of genes & Genes \\
\hline \multicolumn{3}{|l|}{ Upregulated genes } \\
\hline ECM-receptor interaction & 10 & $\begin{array}{l}\text { COL4A1, CD44, COL3A1, COL1A2, LAMC2, COL1A1, COL5A2, } \\
\text { THBS2, COL11A1, COL5A1 }\end{array}$ \\
\hline Pathways in cancer & 18 & $\begin{array}{l}\text { BID, WNT5A, TCF7, COL4A1, IL8, MET, BRCA2, LEF1, CDK6, } \\
\text { MMP1, WNT2, CCND1, VEGFA, SLC2A1, LAMC2, AXIN2, } \\
\text { RUNX1, MYC }\end{array}$ \\
\hline Focal adhesion & 13 & $\begin{array}{l}\text { COL4A1, COL3A1, MET, COL5A2, COL5A1, CCND1, VEGFA, } \\
\text { COL1A2, LAMC2, COL1A1, COL11A1, THBS2, PARVB }\end{array}$ \\
\hline Bladder cancer & 5 & CCND1, IL8 ${ }^{\mathrm{a}}, \mathrm{VEGFA}, \mathrm{MYC}, \mathrm{MMP}^{\mathrm{a}}$ \\
\hline p53 signaling pathway & 6 & BID, CCND1, SERPINE1, CDK6, PMAIP1, PERP \\
\hline Wnt signaling pathway & 9 & $\begin{array}{l}\text { WNT2, WNT5A, TCF7, CCND1, SFRP4a , LEF1, RUVBL1, AXIN2, } \\
\text { MYC }\end{array}$ \\
\hline Thyroid cancer & 4 & TCF7, CCND1, LEF1, MYC \\
\hline Endometrial cancer & 5 & TCF7, CCND1, LEF1, AXIN2, MYC \\
\hline Basal cell carcinoma & 5 & WNT2 $^{\mathrm{a}}$, WNT5A, TCF7, LEF1, AXIN2 \\
\hline Colorectal cancer & 6 & TCF7, CCND1, MET, LEF1, AXIN2, MYC \\
\hline Acute myeloid leukemia & 5 & TCF7, CCND1, LEF1, RUNX1, MYC \\
\hline \multicolumn{3}{|l|}{ Downregulated genes } \\
\hline Cell adhesion molecules & 8 & $\begin{array}{l}\text { NCAM1, CADM1, PVRL3, NLGN4X, NLGN1, NFASC, CNTN1, } \\
\text { NRXN1 }^{\text {a }}\end{array}$ \\
\hline
\end{tabular}

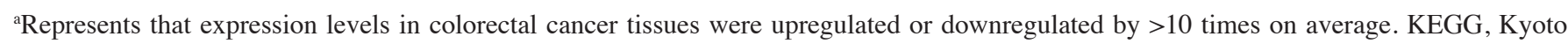
Encyclopedia of Genes and Genomes.

pathways were: i) Extracellular matrix (ECM)-receptor interaction (70 genes); ii) pathways in cancer (99 genes); iii) focal adhesion (86 genes); iv) cytokine-cytokine receptor interaction (56 genes); and v) bladder cancer (14 genes). The downregulated genes were involved in 7 signal transduction pathways, and the top 5 pathways were: i) Axon guidance (10 genes); ii) cell adhesion molecules (8 genes); iii) vascular smooth muscle contraction ( 7 genes); iv) calcium signaling pathway (9 genes); and v) butanoate metabolism (4 genes). Table IV summarizes the genes and pathways associated with colorectal cancer, as filtered out by the KEGG analysis.

\section{Discussion}

Colorectal cancer is one of the most common types of malignant tumors, and its incidence has shown an increasing trend in recent years. The 2012 Annual Report of Cancer Registration in China released by the National Cancer Registry Center (20) shows that the growth rate of the incidence of colorectal cancer in Zhejiang, Shanghai and Jiangsu far exceeded that in Western countries. In Shanghai, the latest cancer surveillance data show that, from the early 1970s to date, the ranking of colorectal cancer has risen from sixth to second among common malignant tumors, with an increase of $\sim 5$ times (21). In the 2013 Ninth Shanghai International Forum of Colorectal Cancer, Professor Qin Xinyu, from Zhongshan Hospital of Fudan University revealed that the epidemiological trend in colorectal cancer in China in the past 20 years has shown a novel change from low-incidence disease to high-incidence disease and the absolute number of morbidity and fatality has exceeded that in the USA in previous years, with a high proportion of young patients (22). The average age of colorectal cancer onset in China is now equal to developed countries. However, similar to numerous other typical gastrointestinal cancers, colorectal cancer has a long time of progression, generally 8 to 10 years, which provides sufficient time for detection and treatment. Through active prevention, early diagnosis, and effective treatment, it is possible to cure this lethal disease (23). Therefore, early detection and diagnosis of colorectal cancer may fundamentally change the outcome of colorectal cancer patients.

cDNA microarrays are a high-throughput, integrated and emerging inspection technology that have been used for investigations at the genomic, transcriptomic and epigenetic levels. In the present study, Human Genome U133 Plus 2.0 Array chips were used to compare 6 pairs of gene expression profiles from colorectal cancer and adjacent normal tissues. In total, 1,183 genes, including 570 that were upregulated and 613 that were downregulated, were identified to be differentially expressed between these two groups. Subsequently, 4 genes that were upregulated (KRT23, COL10A1, COL11A1 and KIAA1199) and 2 genes that were downregulated (SCN7A and EPHA7) in all 6 patients with carcinoma were selected for validation by RT-qPCR. TGFBI and MYC, which are known to be upregulated in cancers (15), served as positive controls. The RT-qPCR results were consistent with the microarray results, which demonstrated the reliability of the microarray results. 
Cluster analysis is one of the commonly used methods in microarray data mining. By calculating the similarities of the data, the unsupervised data obtained from microarrays were classified to cluster similar data. It has been demonstrated (24) theoretically that genes that are classified into one cluster potentially have similar or associated functions; thus, the functions of unknown genes may be deduced by functional association. The heat-map of the cluster analysis in the present study suggested that the expression patterns of the first 6 samples were different from the other 6 samples. The two types of expression patterns among these 12 samples represented two types of samples: The cancer tissues and adjacent normal tissues. These results suggested that the cancer tissues and adjunct normal tissues had different gene expression patterns. Using cDNA microarrays, 1,183 differentially expressed genes were identified between the cancer and normal tissues, which may lay the foundation for subsequent analysis in future studies.

By GO analysis, the upregulated genes were divided into 51 categories, and the downregulated genes were classified into 20 categories, according to different functions. Through KEGG pathway analysis, the upregulated genes were associated with 16 signal transduction pathways and the downregulated genes were associated with 7 signal transduction pathways; these included pathways that are known to be directly associated with the development of colorectal cancer or other cancers, such as ECM-receptor interaction. ECM-receptor interaction was identified to be significantly activated in patients with meningioma (25). Other key pathways that regulate cell adhesion, migration, proliferation and survival were downregulated, and have been associated with numerous tumors, including breast and thyroid carcinoma (26). The p53 signaling pathway was demonstrated to serve a pivotal role by forming a complex with its target genes to suppress tumorigenesis (27), and the Wnt signaling pathway, which is a complex network of protein-protein interactions and a major pathway involved in cancer development and embryogenesis, have been reported to be abnormally regulated in a variety of tumors (28-31). Amongst the pathways associated with the differentially expressed genes in the present study, 6 genes identified to have previously been directly associated with colorectal cancer: T-cell-specific transcription factor 1 (TCF7) (32), cyclin D1 (CCND1) (33), tyrosin-protein kinase met (MET) (34), lymphoid enhancer binding factor 1 (LEF1) (35), axin 2 (AXIN2) (36) and MYC (37). TCF7, CCND1, LEF1, AXIN2 and MYC were all associated with the Wnt signaling pathway. TCF7 and LEF1 regulate transcription initiation by forming a coactivating complex with nuclear $\beta$-catenin. P53 and the nuclear receptor family may inhibit the Wnt signaling pathway by regulating TCF7/LEF1 activation or repression (38). AXIN2 (39) and MYC (40) are the targets in the classic Wnt signaling pathway, which modulates the stability of $\beta$-catenin to regulate cell proliferation and differentiation. CCND1 and MET are the receptors for the hepatocyte growth factor (HGF); the activation of HGF results in the phosphorylation of two MET-adjacent tyrosine residues, which in turn activates multiple signaling pathways, thereby promoting cell division, angiogenesis, tumor cell invasion and metastasis $(41,42)$. CCND1 is a key factor in the regulation of the cell cycle, and its overexpression shortens the G1/S phase, such that cells produce less growth-dependent cytokines, which in turn affects tumor occurrence, development and prognosis (43).

With the current popularity of gene chip technology and rapid development of bioinformatics, more studies are exploring tumor-associated gene signaling pathways. Key gene effectors and signaling pathways involved in the occurrence and development of colorectal cancer have gradually been revealed (44-46). In the present study, a cDNA microarray analysis was performed to screen for genes that were differentially expressed between colorectal cancer and adjacent normal tissues. A KEGG analysis revealed 6 overlapping genes amongst numerous signaling pathways, which have been implicated in the development of a variety of tumors, were also significantly enriched in colorectal cancer tissues. These 6 genes, which have been closely associated with the development of colorectal cancer, were involved in the regulation of the cell cycle, adhesion, metabolism, proliferation and differentiation; thus, their dysregulation may be involved in the process of tumor development. Further research on these genes and the implicated signaling pathways may shed new light on the pathogenesis of colorectal cancer.

\section{Acknowledgements}

The present study was supported by the National Natural Science Foundation of China (grant no. 81072152).

\section{References}

1. Nordlinger B, Sorbye H, Glimelius B, Poston GJ, Schlag PM, Rougier P, Bechstein WO, Primrose JN, Walpole ET, FinchJones M, et al: Perioperative chemotherapy with FOLFOX4 and surgery versus surgery alone for resectable liver metastases from colorectal cancer (EORTC Intergroup trial 40983): A randomised controlled trial. Lancet 371: 1007-1016, 2008.

2. Ferlay J, Soerjomataram I, Dikshit R, Eser S, Mathers C, Rebelo M, Parkin DM, Forman D and Bray F: Cancer incidence and mortality worldwide: Sources, methods and major patterns in GLOBOCAN 2012. Int J Cancer 136: E359-E386, 2015.

3. Jemal A, Bray F, Center MM, Ferlay J, Ward E and Forman D: Global cancer statistics. CA Cancer J Clin 61: 69-90, 2011.

4. Torre LA, Bray F, Siegel RL, Ferlay J, Lortet-Tieulent J and Jemal A: Global cancer statistics, 2012. CA Cancer J Clin 65: 87-108, 2015.

5. Wan D: Epidemiology and prevention of colorectal cancer. Chin J Surg Integ Tradit West Med 17: 3-7, 2012.

6. Galizia G, Gemei M, Del Vecchio L, Zamboli A, Di Noto R, Mirabelli P, Salvatore F, Castellano P, Orditura M, De Vita F, et al: Combined CD133/CD44expression as a prognostic indicator of disease-free survival in patientswith colorectal cancer. Arch Surg 147: 18-24, 2012.

7. The changing face of UK primary cancer care: Lancet Oncol 2: 649,2001

8. Speights VO, Johnson MW, Stoltenberg PH, Rappaport ES, Helbert B and Riggs M: Colorectal cancer: Current trends in initial clinical manifestations. South Med J 84: 575-578, 1991.

9. Summerton N: Symptoms of possible oncological significance: Separating the wheat from the chaff. BMJ 325: 1254-1255, 2002.

10. Flashman K, O'Leary DP, Senapati A and Thompson MR: The department of health's 'two week standard' for bowel cancer: Is it working? Gut 53: 387-391, 2004.

11. Knottnerus JA, Knipschild PG and Sturmans F: Symptoms and selection bias: The influence of selection towards specialist care on the relatioship between symptoms and diagnosis. Theor Med 10: 67-81, 1989.

12. LaPointe LC, Pedersen SK, Dunne R, Brown GS, Pimlott L, Gaur S, McEvoy A, Thomas M, Wattchow D, Molloy PL, et al: Discovery and validation of molecular biomarkers for colorectal adenomas and cancer with application to blood testing. PLoS One 7: e29059, 2012. 
13. Yi JM, Dhir M, Guzzetta AA, Iacobuzio-Donahue CA, Heo K, Yang KM, Suzuki H, Toyota M, Kim HM, Ahuja N, et al: DNA methylation biomarker candidates for early detection of colon cancer. Tumour Biol 33: 363-72, 2012.

14. Gautier L, Cope L, Bolstad BM and Irizarry RA: Affy-analysis of Affymetrix GeneChip data at the probe level. Bioinformatics 20: 307-315, 2004.

15. Wright JB, Brown SJ and Cole MD: Upregulation of c-MYC in cis through a large chromatin loop linked to a cancer risk-associated single-nucleotide polymorphism in colorectal cancer cells. Mol Cell Biol 30 1411-1420, 2010.

16. Livak KJ and Schmittgen TD: Analysis of relative gene expression data using real-time quantitative PCR and the 2(-Delta Delta C(T)) method. Methods 25: 402-408, 2001

17. de Hoon MJ, Imoto S, Nolan J and Miyano S: Open source clustering software. Bioinformatics 20: 1453-1454, 2004.

18. Maitra A, Adsay NV, Argani P, Iacobuzio-Donahue C, De Marzo A, Cameron JL, Yeo CJ and Hruban RH: Multicomponent analysis of the pancreatic adenocarcinoma progression model using a pancreatic intraepithelial neoplasia tissue microarray. Mod Pathol 16: 902-912, 2003.

19. Huber W, Von Heydebreck A and Vingron M: Analysis of microarray gene expression data. In: Handbook of Statistical Genetics (2nd ed). John Wiley \& Sons (NJ, USA), pp37-53, 2003.

20. National Cancer Centre, Bureau for Disease Control and Prevention of Health Ministry: The 2011 Chinese cancer registry annual report. Military Medical Science Press 17, Beijing, pp53-57,58, 2012 (In Chinese)

21. Chen JG, Zhu J, Parkin DM, Zhang YH, Lu JH, Zhu YR and Chen TY: Trends in the incidence of cancer in Qidong, China, 1978-2002. Int J Cancer 119: 1447-1454, 2006.

22. Xu J, Qin X, Wang J, Zhang S, Zhong Y, Ren L, Wei Y, Zeng S, Wan D, Zheng S, et al: Chinese guidelines for the diagnosis and comprehensive treatment of hepatic metastasis of colorectal cancer. J Cancer Res Clin Oncol 137: 1379-1396, 2011.

23. Fearon ER: Molecular genetics of colorectal cancer. Annu Rev Pathol 6: 479-507, 2011.

24. Quackenbush J: Computational analysis of microarray data. Nat Rev Genet 2: 418-427, 2001.

25. Wang X, Gong Y, Wang D, Xie Q, Zheng M, Zhou Y, Li Q, Yang Z, Tang H, Li Y, et al: Analysis of gene expression profiling in meningioma: Deregulated signaling pathways associated with meningioma and EGFL6 overexpression in benign meningioma tissue and serum. PLoS One 7: e52707, 2012.

26. Fang XQ, Liu XF, Yao L, Chen CQ, Gu ZD, Ni PH, Zheng XM and Fan QS: Somatic mutational analysis of FAK in breast cancer: A novel gain-of-function mutation due to deletion of exon 33. Biochem Biophys Res Commun 443: 363-369, 2014

27. Liu J, Zhang $C$ and Feng Z: Tumor suppressor p53 and its gain-of-function mutants in cancer. Acta Biochim Biophys Sin (Shanghai) 46: 170-179, 2014.

28. Colli LM, Saggioro F, Serafini LN, Camargo RC, Machado HR, Moreira AC, Antonini SR and de Castro M: Components of the canonical and non-canonical Wnt pathways are not mis-expressed in pituitary tumors. PLoS One 8: e62424, 2013.

29. Cai Y, Cai T and Chen Y: Wnt pathway in osteosarcoma, from oncogenic to therapeutic. J Cell Biochem 115: 625-631, 2014.

30. Wang SH, Li N, Wei Y, Li QR and Yu ZP: $\beta$-catenin deacetylation is essential for WNT-induced proliferation of breast cancer cells. Mol Med Rep 9: 973-978, 2014.
31. Krausova $\mathbf{M}$ and Korinek V: Wnt signaling in adult intestinal stem cells and cancer. Cell Signal 26: 570-579, 2014.

32. Waterman ML: Lymphoid enhancer factor/T cell factor expression in colorectal cancer. Cancer Metastasis Rev 23: 41-52, 2004

33. Tetsu $\mathrm{O}$ and McCormick F: Beta-catenin regulates expression of cyclin D1 in colon carcinoma cells. Nature 398: 422-426, 1999.

34. Emaduddin M, Bicknell DC, Bodmer WF and Feller SM: Cell growth, global phosphotyrosine elevation, and c-Met phosphorylation through Src family kinases in colorectal cancer cells. Proc Natl Acad Sci USA 105: 2358-2362, 2008.

35. Kriegl L, Horst D, Reiche JA, Engel J, Kirchner T and Jung A: LEF-1 and TCF4 expression correlate inversely with survival in colorectal cancer. J Transl Med 8: 123, 2010.

36. Liu W, Dong X, Mai M, Seelan RS, Taniguchi K, Krishnadath KK, Halling KC, Cunningham JM, Boardman LA, Qian C, et al: Mutations in AXIN2 cause colorectal cancer with defective mismatch repair by activating beta-catenin/TCF signalling. Nat Genet 26: 146-147, 2000.

37. He TC, Sparks AB, Rago C, Hermeking H, Zawel L, da Costa LT, Morin PJ, Vogelstein B and Kinzler KW: Identification of c-MYC as a Target of the APC Pathway. Science 281: 1509-1512, 1998.

38. Mao CD and Byers SW: Cell-context dependent TCF/LEF expression and function: Alternative tales of repression, de-repression and activation potentials. Crit Rev Eukaryot Gene Expr 21: 207-236, 2011.

39. Wang FW, Wen L, Zhu SW, Yao Q, Cai YM and Ma G: Mechanism of Wnt signaling pathway regulation by a truncated mutant of Axin2 in colorectal cancer. Cancer 26: 1041-1046, 2007 (In Chinese).

40. van de Wetering M, Sancho E, Verweij C, de Lau W, Oving I, Hurlstone A, van der Horn K, Batlle E, Coudreuse D, Haramis AP, et al: The beta-catenin/TCF-4 complex imposes a crypt progenitor phenotype on colorectal cancer cells 111: 241-250, 2002.

41. Wang Y and Zheng T: Screening of hub genes and pathways in colorectal cancer with microarray technology. Pathol Oncol Res 20: 611-618, 2014.

42. Nishimura $Y$, Takiguchi S, Ito $S$ and Itoh K: Evidence that depletion of the sorting nexin 1 by siRNA promotes HGF-induced MET endocytosis and MET phosphorylation in a gefitinib-resistant human lung cancer cell line. Int J Oncol 44: 412-426, 2014.

43. Naushad SM, Reddy CA, Kumaraswami K, Divyya S, Kotamraju S, Gottumukkala SR, Digumarti RR and Kutala VK: Impact of hyperhomocysteinemia on breast cancer initiation and progression: Epigenetic perspective. Cell Biochem Biophys 68: 397-406, 2014.

44. Han L, Wu Z and Zhao Q: Revealing the molecular mechanism of colorectal cancer by establishing LGALS3-related protein-protein interaction network and identifying signaling pathways. Int J Mol Med 33: 581-588, 2014.

45. Takahashi Y,Sawada G, Kurashige J,Uchi R, Matsumura T, Ueo H, Takano Y, Eguchi H, Sudo T, Sugimachi K, et al: Amplification of PVT-1 is involved in poor prognosis via apoptosis inhibition in colorectal cancers. Br J Cancer 110: 164-171, 2014.

46. Gantt GA, Chen Y, Dejulius K, Mace AG, Barnholtz-Sloan J and Kalady MF: Gene expression profile is associated with chemoradiation resistance in rectal cancer. Colorectal Dis 16: 57-66, 2014. 